\title{
Extra-cardiac coronary communication to the right coronary artery
}

\author{
Rabih R. Azar ${ }^{1}$, Zena Kadri ${ }^{1}$, Abdelkarim Najib ${ }^{2}$, Antonio H. Frangieh ${ }^{3}$ \\ ${ }^{1}$ Hotel Dieu de France Hospital, St. Joseph University, Beirut, Lebanon \\ ${ }^{2}$ Lebanon Heart Hospital, Tripoli, Lebanon \\ ${ }^{3}$ Deutsches Herzzentrum München, Technische Universität München, Munich, Germany
}

\section{Introduction}

Collateral circulation from a normal to a severely narrowed coronary artery are common and explain why necrosis did not occur in a myocardial segment that receives its supply from an occluded coronary artery. However, communications between coronary arteries and extra-cardiac systemic vessels (extra-cardiac collaterals) are rare and have been mainly demonstrated by post-mortem studies $[1,2]$. Their functional significance is unclear.

Presented here, are the coronary angiograms of 2 patients with functionally significant extracardiac communications/collaterals arising from a systemic artery in the thorax and connecting with the right coronary artery (RCA).

\section{Case 1}

The patient is a 66 -year-old male admitted with congestive heart failure. Electrocardiogram (ECG) revealed left ventricular hypertrophy with secondary ST segment and T wave abnormalities. Cardiac echocardiography revealed a dilated cardiomyopathy. Coronary angiography was performed to exclude coronary artery disease as a cause of his cardiomyopathy. All coronary arteries were free of significant disease. On injection of the RCA an approximately $1.5 \mathrm{~mm}$ diameter vessel arising from the distal RCA (Fig. 1A) extending all the way up to the chest (Fig. 1B; Supplementary Video 1 - see journal website) was visualized. That vessel is divided into

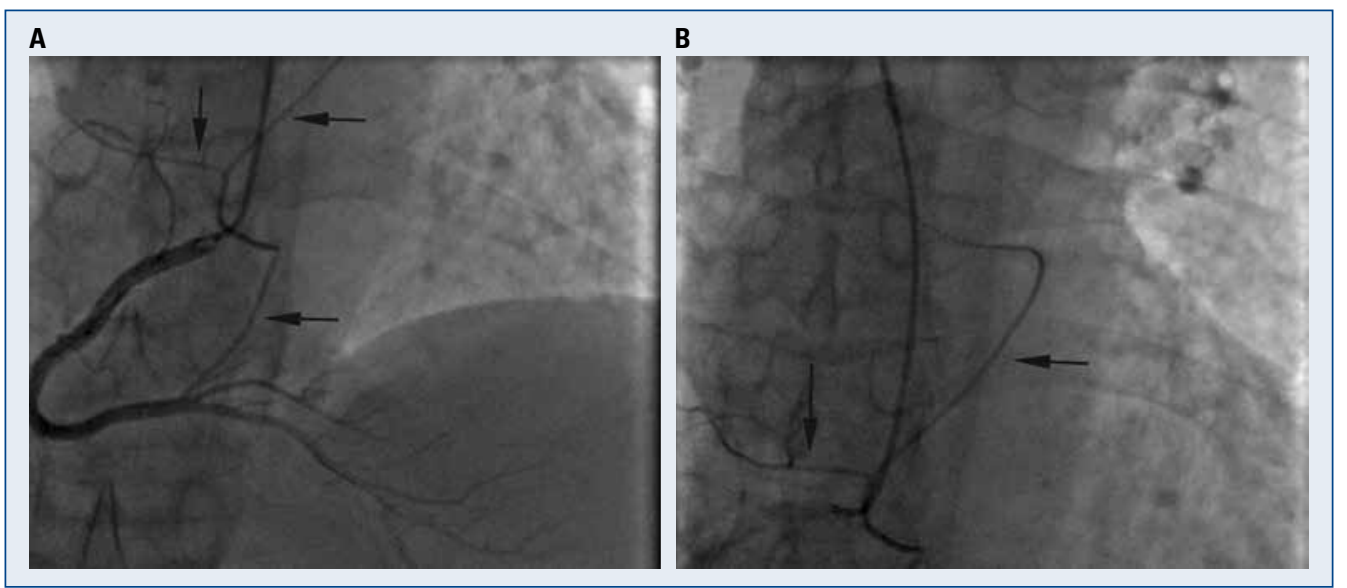

Figure 1. Case 1 angiogram; A. Angiography of a normal right coronary artery with visualization of a $1.5 \mathrm{~mm}$ vessel (arrows) arising from the distal segment and extending all the way up; B. Continuation of the extra-cardiac communication (arrows), well above the heart into the thorax.

Address for correspondence: Rabih R. Azar, MD, MPH, FACC, Division of Cardiology, Hotel Dieu de France Hospital, Achrafieh, Beirut, Lebanon, tel: +961 1615300 ext. 9408, fax: +961 1615300 ext. 9402, e-mail: razar@usj.edu.lb Received: 06.05.2016 Accepted: 23.11.2016 


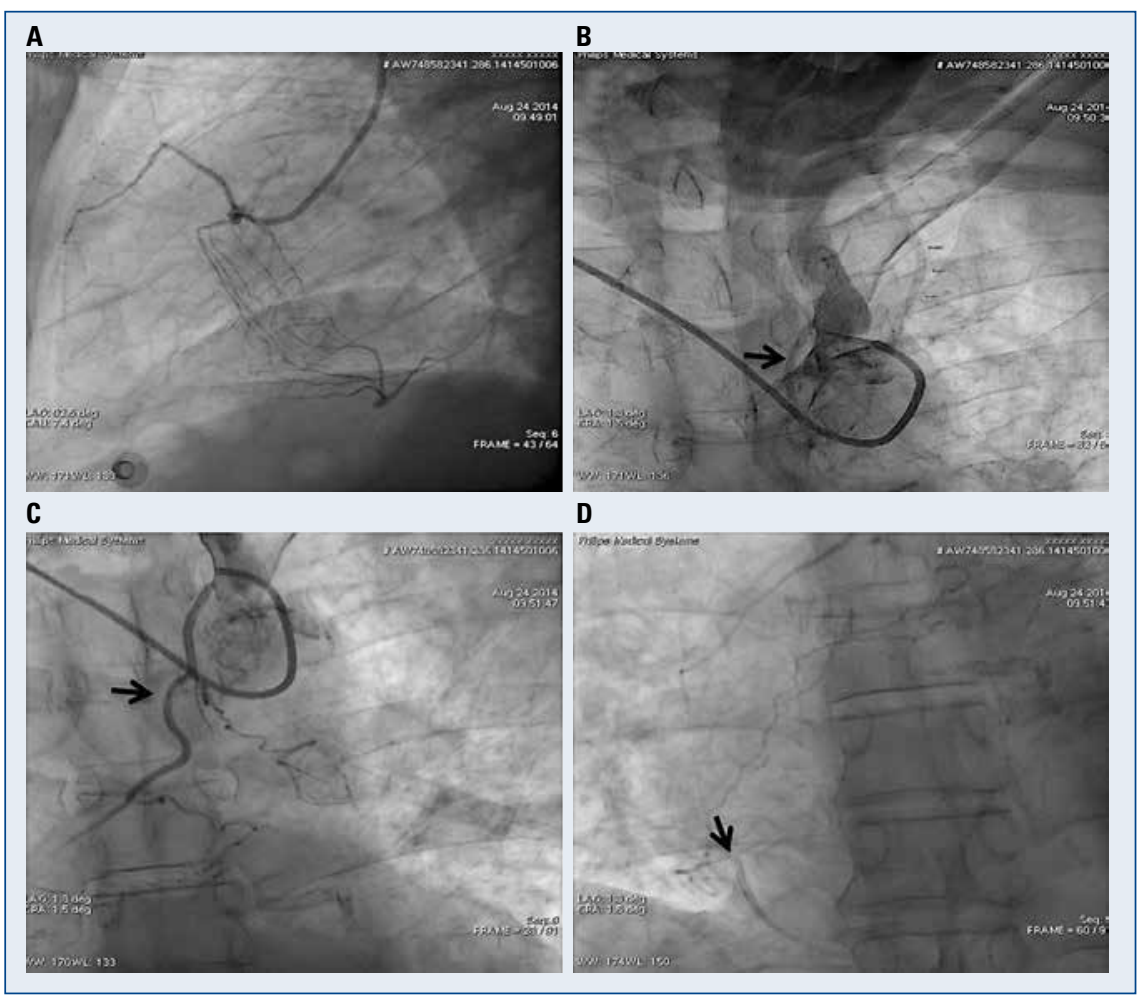

Figure 2. Case 2 angiogram; A. Right coronary artery angiogram showing proximal occlusion of that vessel; B. Injection in the left subclavian artery with visualization of a branch that runs downward (arrow); C. Continuation of the cine run showing division of that branch (arrow) into several smaller vessels; D. One of the collateral branches (arrow) connects with and fills the distal right coronary artery.

two distinct branches. The arteries to which these two branches were connected could not be visualized, however, they were clearly above the heart. These vessels represent communications between a coronary artery and extra-cardiac vessels that were functional despite the fact that the RCA was free of significant atherosclerosis and had normal flow.

\section{Case 2}

The patient is a 70-year-old male who was admitted with a non-ST elevation myocardial infarction and pulmonary edema. His admission ECG showed atrial fibrillation with 1-2 mm downslopping ST depression in the antero-lateral leads. He was rapidly stabilized with anti-platelet therapy (aspirin and ticagrelor), enoxaparin, nitrates and diuretics. ST segment depression improved and he did not develop $\mathrm{Q}$ waves. There was minor elevation in cardiac troponin.

Cardiac echocardiography revealed global hypokinesis with left ventricular ejection fraction of $40 \%$. Coronary angiography showed tight proximal left anterior descending artery (LAD) stenosis with
Thrombolysis in Myocardial Infarction (TIMI) III flow, occlusion of the circumflex artery after the take off of the first marginal branch and proximal occlusion of RCA (Fig. 2A). The interventional cardiologist elected to visualize the left internal mammary artery in order to decide if it was suitable for coronary artery bypass graft surgery. A non-selective injection was made in the left subclavian artery. It demonstrated the presence of an artery that arises from the right side of the subclavian (Fig. 2B; Supplementary Video 2 - see journal website) extends down toward the right side of the chest providing several smaller branches throughout its course (Fig. 2C; Supplementary Video 2 - see journal website); one of these branches, most likely a pericardial branch, connects with the mid-distal RCA and completely fills that vessel (Fig. 2D; Supplementary Video 2 - see journal website). Flow in the distal RCA was normal.

\section{Comments}

Extra-cardiac collaterals to the coronary arteries are not rare and have been reported in 10-20\% 
of cases, but they are usually small and their flow is limited [3].

According to available literature, this is the first reported case of a functional extra-cardiac communication to a normal RCA. That communication was significant in size and had normal flow. Injection of the RCA allowed complete visualization of that extra-cardiac vessel.

In addition, case 2 is the first reported case of extra-cardiac collaterals emerging from the left subclavian artery and filling an occluded RCA. Prior observations have reported extra-cardiac collaterals arising from the left and right internal mammary arteries to LAD and to RCA and from bronchial arteries to RCA through pericardial branches [3, 4].

This reported case is interesting for several reasons: It clearly demonstrates the presence of extra-cardiac collaterals. It supports prior observations that these collaterals are usually present before the development of coronary artery disease. It also shows that in some cases, these collaterals are capable of normal flow, even in the absence of obstructive disease in the native vessel. Case 2 demonstrates their importance once obstruction occurs in the native vessel. The flow they carry allows significant perfusion of the distal coronary artery, limiting the amount of necrosis.

Because these collaterals are extra-cardiac, they are frequently not diagnosed. Their presence should be considered in patients with occluded native coronaries who do not have symptoms or signs of ischemia and/or evidence of myocardial necrosis.

Conflict of interest: None declared

\section{References}

1. Moberg A. Anastomoses between extracardiac vessels and coronary arteries. 1. Via bronchial arteries. Acta Radiol (Diagn). 1967; 6(2): 177-192, doi: 10.1177/028418516700600209.

2. Moberg A. Anastomoses between extracardiac vessels and coronary arteries. II. Via internal mammary arteries. Post-mortem angiographic study. Acta Radiol Diagn (Stockh). 1967; 6(3): 263-272, indexed in Pubmed: 6025072.

3. Loukas M, Hanna M, Chen J, et al. Extracardiac coronary arterial anastomoses. Clin Anat. 2011; 24(2): 137-142, doi: 10.1002/ /ca.21088, indexed in Pubmed: 21322035.

4. Kajinami K, Takekoshi N, Yoshio H. Internal mammary-to-coronary artery communication in a patient with occluded right coronary artery. Am Heart J. 1993; 125(5): 1428-1430, indexed in Pubmed: 8480598. 